\title{
Usabilidad y aceptabilidad del programa de tratamiento autoaplicado a través de Internet para la fobia a volar SIN MIEDO Airlines: resultados de un estudio piloto
}

\author{
Daniel Campos Bacas \\ camposd@uji.es \\ ADRIANA MIRA PASTOR \\ miraa@uji.es \\ DIANA CASTILLA \\ castilla@uji.es \\ JUANA BRETÓN LÓPEZ \\ breton@uji.es \\ SolEdAd QUERO CASTELLANO \\ squero@uji.es
}

\section{Resumen}

Introducción: El tratamiento de elección para las fobias es la exposición en vivo, mostrando su eficacia en distintos estudios. No obstante, existen limitaciones en su aplicación en relación al acceso y aceptación por parte de clínicos y pacientes. Los tratamientos aplicados a través del ordenador pueden ser una alternativa eficaz para mejorar esta técnica. Internet es una novedosa forma de aplicar tratamientos psicológicos eficaces reduciendo costes y facilitando el acceso. Hasta la fecha, no existen estudios que exploren la aceptabilidad de un tratamiento autoaplicado vía Internet para la fobia a volar (FV). El objetivo de este estudio es ofrecer datos sobre usabilidad y aceptabilidad de SIN MIEDO Airlines en un estudio piloto. Método: La muestra se compuso de 4 participantes $(M=34,5$ años; $D T=11,1)$ residentes en España $(N=3)$ y Colombia $(\mathrm{N}=1)$. Al finalizar el tratamiento, contestaron el cuestionario de usabilidad y aceptabilidad (CUA) formado por 10 ítems para evaluar: la rapidez de aprendizaje, la capacidad de uso, la orientación, la eficiencia, el modelo ecológico, la facilidad de las instrucciones, el control y la visibilidad, la intención de uso, la utilidad y la facilidad percibida. Resultados: Los resultados mostraron puntuaciones elevadas en el CUA ( $M=93,8$; $D T=7,5)$ en un rango de 85 a 100, estableciéndose en la categoría «mejor imaginable» de acuerdo con Bangor, Kortum y Miller (2008, 2009). Conclusión: Los datos obtenidos a partir del estudio piloto reflejan una buena valoración de los participantes respecto a la usabilidad y aceptabilidad de SIN MIEDO Airlines. Estos resultados podrían tener repercusiones en la mejora del tratamiento de la $\mathrm{FV}$, concretamente en relación a la técnica de la exposición en vivo.

Palabras clave: fobia a volar, tratamiento autoaplicado, Internet, usabilidad. 


\section{Abstract}

Introduction: The treatment of choice for specific phobias is in vivo exposure. Despite the proven efficacy of this technique, it is linked to a number of limitations in its implementation among patients and therapists. Computer-assisted exposure programs could be a useful tool in order to improve this technique. Moreover, the Internet is a novel way to apply effective psychological treatments reducing costs and improving the access to the treatment. However, to our knowledge, no other study investigates the acceptability of a self-applied treatment via the Internet for flying phobia (FP). The aim of this work is to provide data regarding the usability and acceptability of NO-FEAR Airlines in a pilot study. Method: The sample was composed of four participants (mean age $=34.5$; $\mathrm{SD}=11.1)$ residents in Spain $(\mathrm{N}=3)$ and Colombia $(\mathrm{N}=1)$. After the treatment, participants completed the Usability and Acceptability Questionnaire (UAQ) composed by 10 items in order to assess: speed learning, usability, orientation, efficiency, ecological model, instructions easiness, control and visibility, intention to use, usefulness and perceived ease. Results: Results showed high UAQ scores ( $M=93.8$; $S D=7.5)$ from 85 to 100 , resulting in the best imaginable category according to Bangor, Kortum and Miller (2008, 2009). Conclusion: NO-FEAR Airlines was well assessed in terms of usability and acceptability. Results could have implications to improve the FP treatment, specifically, the in vivo exposure technique.

Keywords: flying phobia, self-applied treatment, Internet, usability.

\section{Introducción}

Una de las fobias más presentes en nuestra sociedad es la fobia a volar (FV), con una prevalencia de alrededor del 2,5 (Oakes y Bor, 2010). Aproximadamente un $25 \%$ de la población adulta experimenta un nivel significativo de ansiedad cuando tiene que volar, alrededor del $10 \%$ de la población general no vuela como consecuencia de la intensidad de su miedo y un $20 \%$ depende del alcohol o los tranquilizantes para poder volar (Botella, Osma, GarcíaPalacios, Quero y Baños, 2004). Las implicaciones personales causadas por la FV son muy diversas, dependiendo de las necesidades familiares, laborales y de ocio de cada persona, así como de su ubicación geográfica (Oakes y Bor, 2010). En este sentido, padecer este problema puede limitar las oportunidades profesionales y las opciones de ocio, alterar las relaciones personales, además de ir acompañado de vergüenza y malestar emocional cuando la persona se enfrenta a la idea de volar (Bor, 2007; Iljon Foreman, Bor y Van Gerwen, 2006).

En la actualidad, el tratamiento de elección para las fobias es la exposición en vivo (Nathan y Gorman, 2007), rondando su porcentaje de éxito el 80 \% (Capafons, 2001). No obstante, existen limitaciones en su aplicación en relación al acceso y aceptación del mismo por parte de clínicos y pacientes. En primer lugar, la mayoría de las personas que padecen fobia nunca buscan tratamiento, solamente el $7,8 \%$ lo busca y tan solo un $0,8 \%$ de los pacientes recibe un tratamiento específico para este trastorno (Boyd y cols., 1990; Mackenzie, Reynolds, Cairney, Streiner y Sareen, 2012; Stinson y cols., 2007). Esto puede deberse a diversas razones: a la falta de terapias basadas en la evidencia ofrecidas en los distintos sistemas de salud, las largas listas de espera o bien a la falta de un entrenamiento adecuado para que los terapeu- 
tas apliquen la técnica de exposición (Kazdin y Blase, 2011; Kazdin y Rabitt, 2013; Kazdin, 2014). Además, aproximadamente un $25 \%$ de las personas rechaza el tratamiento cuando son informadas acerca del procedimiento que se va a seguir o lo abandonan cuando ya lo han comenzado (por ejemplo, García-Palacios, Botella, Hoffman y Fabregat, 2007; García-Palacios, Hoffman, See, Tsay y Botella, 2001), rondando las tasas de abandono el 20,6 \% (Olatunji, Deacon y Abramowitz, 2009).

También existen problemas con la aceptación de la terapia de exposición por parte de los terapeutas (Moritz, Hoffman, Herbert y Schare, 2013; Richard y Gloster, 2007) ya que algunos autores la han llegado a considerar inhumana y éticamente inapropiada (Deacon y Farrell, 2013; Olatunji, Deacon y Abramowitz, 2009). En algunos casos, esta técnica presenta también otras limitaciones, como la falta de confidencialidad o los costes asociados cuando debe realizarse fuera de la consulta (Kazdin y Rabitt, 2013; Konnopka, Leichsenring, Leibing y König, 2009). Otra dificultad especialmente relevante en la FV es el difícil acceso al estímulo temido (por ejemplo, aeropuerto o avión) (Rothbaum, Hodges, Smith, Lee y Price, 2000).

En este sentido, de acuerdo con Kazdin (2014), es necesario desarrollar nuevos modelos para aplicar los tratamientos basados en la evidencia para tratar de reducir el impacto de los trastornos mentales. En el caso concreto de las fobias, es necesario explorar formas de mejorar la terapia de exposición para aumentar el número de pacientes que se puedan beneficiar de la misma, lo que pasa por mejorar la aceptación de este tratamiento y aplicar la exposición con una menor presencia del terapeuta. Las tecnologías de la información y la comunicación (TIC), como los programas de tratamiento de realidad virtual (RV) y los tratamientos asistidos por ordenador, pueden ser una alternativa eficaz para mejorar esta técnica. Estas herrramientas pueden favorecer tanto la accesibilidad a la terapia como el grado de aceptación de la misma por parte de los pacientes al no generar niveles de ansiedad tan aversivos como la exposición en vivo (García-Palacios y cols., 2007; Quero y cols., 2014).

Distintos estudios han mostrado la eficacia de la RV para el tratamiento de la FV (Baños, Botella, Perpiñá y Quero, 2001; Botella y cols., 2004; Rothbaum y cols., 2010). En esta línea, Tortella-Feliu y cols. (2011) llevaron a cabo un estudio controlado que comparaba un programa de RV (Botella y cols., 2004) con un programa asistido por ordenador para aplicar la exposición (aplicado por el terapeuta vs. autoaplicado) (Computer Assisted Fear of Flight Treatment, CAFFT, Bornas, Fullana, Tortella-Feliu, Llabrés y García de la Banda, 2001). Los resultados mostraron que las tres intervenciones fueron igual de eficaces para el tratamiento del miedo a volar en el pos-tratamiento y en el seguimiento al año. Estos resultados ponen de manifiesto que intervenciones menos costosas y con menor implicación del terapeuta son igualmente eficaces en la reducción de la FV.

En terminos de aceptación, todos los participantes valoraron las intervenciones como lógicas, satisfatorias, útiles y no muy aversivas antes del tratamiento, aunque se encontraron diferencias significativas en cuanto a la valoración de la lógica del tratamiento. Concretamente, los participantes de la condición de RV mostraron una mejor valoración en comparación con el grupo que recibió el tratamiento asistido por ordenador de forma autoaplicada. Después de recibir el tratamiento, los participantes continuaron valorando los tratamientos como muy lógicos, satisfactorios, útiles y no muy aversivos. De nuevo, el grupo de RV valoró como más lógico el tratamiento frente al grupo de tratamiento asistido por ordenador (autoaplicado). Además, el grupo de RV estaba más satisfecho en comparación con la condición que recibió el CAFFT (tanto aplicado por el terapeuta como autoaplicado). Sin embargo, estas diferencias desaparecieron en el seguimiento del año.

A partir de estos resultados positivos, los grupos de investigación liderados por Cristina Botella (LabPsiTec), Mariano Alcañíz (Labhuman) y Jordi Llabrés (Labcds) han desarrollado conjuntamente una versión mejorada del programa CAFFT de tratamiento de la FV (SIN MIEDO 
Airlines) para ser totalmente autoaplicada a través de Internet (sin necesidad del apoyo del terapeuta), desde casa y en un ordenador personal. Internet es una herramienta que ha demostrado ser un modo eficaz de aplicar tratamientos psicológicos en una variedad de problemas psicológicos y condiciones médicas (Anderson, 2016; Andrews, Newby y Williams, 2015). No obstante, hasta la fecha no existe ningún estudio que explore la utilidad, en términos de eficacia y aceptación, de un programa autoaplicado a través de Internet para la FV. El objetivo de este estudio es ofrecer datos sobre usabilidad y aceptabilidad de SIN MIEDO Airlines en un estudio piloto.

\section{Método}

\section{Participantes}

La muestra se compuso de 4 participantes ( 3 mujeres y 1 hombre) residentes en España $(N=3)$ y Colombia $(N=1)$. La media de edad fue de $34,5(D T=11,1)$ en un rango de 25 a 50 años. En cuanto al nivel de estudios, el $50 \%$ de los participantes contaba con estudios secundarios mientras que el otro $50 \%$ con estudios universitarios. De los 4 participantes, 2 estaban casados, 1 era soltero y 1 separado. Todos los participantes, cumplían los criterios diagnósticos de fobia específica (fobia a volar) según el DSM-V (APA, 2013).

\section{Instrumentos}

Cuestionario de usabilidad y aceptabilidad (CUA) (Labpsitec, 2015). Es un instrumento ad hoc compuesto por 10 ítems para evaluar el grado de acuerdo y desacuerdo del usuario ( 0 = totalmente en desacuerdo y $4=$ totalmente de acuerdo) respecto a la usabilidad y aceptabilidad del sistema. Concretamente se evalúa: la rapidez de aprendizaje, la capacidad de uso, la orientación, la eficiencia, el modelo ecológico, la facilidad de las instrucciones, el control y la visibilidad, la intención de uso, la utilidad y la facilidad percibida. La puntuación total del CUA oscila entre 0 y 100 . Este cuestionario está basado en la escala de usabilidad propuesta por Bangor, Kortum y Miller (2008, 2009).

SIN MIEDO Airlines es un programa de tratamiento totalmente autoaplicado a través de Internet para la FV (www.fobiavolar.es) que permite a las personas con miedo a volar exponerse a imágenes y sonidos reales relacionadas con su miedo en un ordenador entándar (Quero y cols., 2015).

Este programa sigue una perspectiva de tratamiento cognitivo-comportamental (TCC) formado por tres componentes básicos: la psicoeducación, la exposición y el sobreaprendizaje, siendo el componente principal la técnica de exposición.

a) Psicoeducación. En el primer componente se ofrece información sobre en qué va a consistir el programa así como información específica sobre la FV: a cuánta gente afecta el problema, a qué tipo de personas afecta, cuáles son los componentes fisiológico, cognitivo y conductual (o evitación) y cómo empieza, se mantiene y se soluciona el problema. En este apartado se incluye texto con información relevante para el tratamiento de la FV acompañado de viñetas con ilustraciones para que el contenido terapéutico resulte más atractivo al paciente.

b) Esposición. SIN MIEDO Airlines ofrece distintos escenarios para que el paciente pueda exponerse desde casa a aquellas situaciones a las que teme: 1) preparativos, 2) 
aeropuerto, 3) despegue, 4) vuelo, 5) aterrizaje y 6) noticias de accidentes aéreos. EI programa presenta los escenarios de más fácil a más dificil (de menor ansiedad a mayor) realizando una jerarquía a partir de las respuestas del participante al cuestionario de miedo a volar (Bornas, Tortella-Feliu, García de la Banda, Fullana y Llabrés, 1999).

c) Sobre-aprendizaje. Al finalizar la exposición a los seis escenarios, el usuario puede elegir realizar una exposición adicional (fase de sobreaprendizaje) a cada una de las seis secuencias, pero con un mayor grado de dificultad ya que, en esta ocasión, se simularán las condiciones de tormenta y turbulencias en los mismos escenarios. Este componente tiene como objetivo repasar algunas de las situaciones y afianzar los logros alcanzados.

\section{Procedimiento}

Todos los participantes completaron el screening y una evaluación telefónica antes de ser asignados al azar a dos períodos de línea base (9 y 12 días). Pasado el tiempo de línea base, todos completaron el tratamiento autoaplicado SIN MIEDO Airlines a través de Internet. La duración del tratamiento dependió del ritmo de cada participante con un máximo de 6 semanas. Después del tratamiento, todos los participantes completaron el CUA junto con el resto del protocolo de la evaluación telefónica.

\section{Resultados}

Los resultados revelaron puntuaciones elevadas en la puntuación total del CUA ( $M=3,8$; DT $=7,5$ ) en un rango de 85 a 100, estableciéndose en la categoría «mejor imaginable», según la clasificación propuesta por Bangor y cols. (2008) (véase la figura 1).

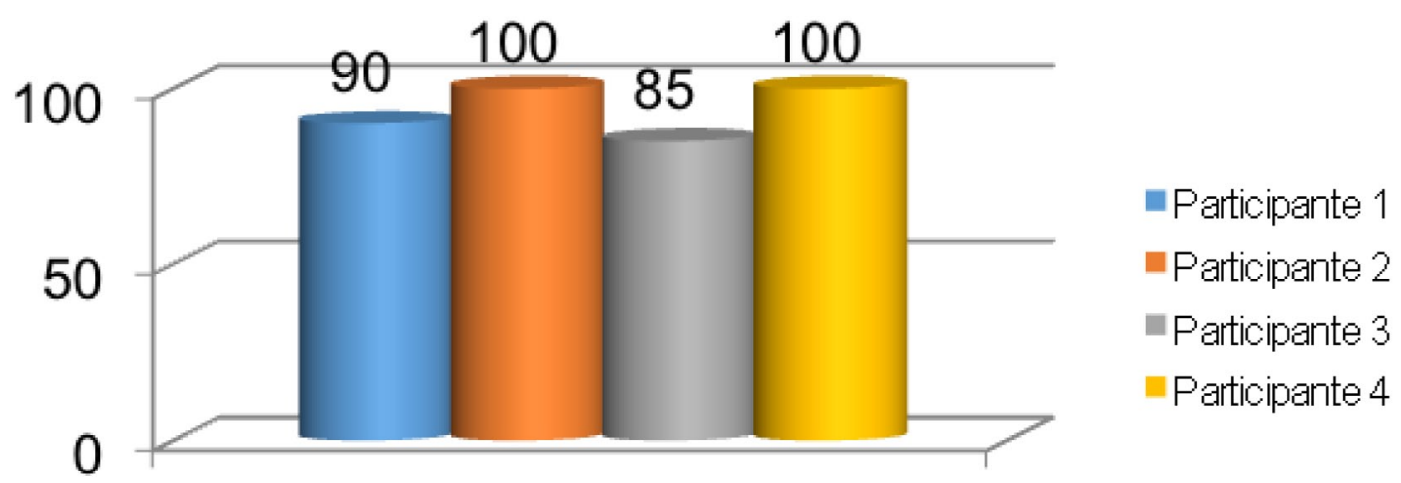

\section{CUA TOTAL}

Figura 1. Puntuación total de cada participante en el CUA 
Todos los participantes informaron puntuaciones elevadas en todos los ítems del CUA, tal y como se puede observar en la tabla 1.

Tabla 1

Media, desviación típica, mínimo y máximo de las puntuaciones en los ítems del CUA

\begin{tabular}{|c|c|c|c|}
\hline ITEM & MEDIA (DT) & MÍNIMO & MÁXIMO \\
\hline $\begin{array}{l}\text { 1. Pienso que la mayoría de las personas podrían aprender } \\
\text { muy rápidamente a utilizar SIN MIEDO Airlines. }\end{array}$ & $3,75(0,5)$ & 3 & 4 \\
\hline $\begin{array}{l}\text { 2. Me he sentido seguro de mí mismo (capaz) utilizando SIN } \\
\text { MIEDO Airlines. }\end{array}$ & $3,75(0,5)$ & 3 & 4 \\
\hline $\begin{array}{l}\text { 3. En general, he sabido qué tenía que hacer en cada mo- } \\
\text { mento. Por ejemplo, cuando he querido pulsar un botón } \\
\text { concreto he sabido cómo hacerlo y lo he conseguido. }\end{array}$ & $3,75(0,5)$ & 3 & 4 \\
\hline $\begin{array}{l}\text { 4. Una vez que he aprendido a usar SIN MIEDO Airlines he } \\
\text { podido realizar las tareas rápidamente. }\end{array}$ & $\begin{array}{c}4 \\
(0,0)\end{array}$ & 4 & 4 \\
\hline $\begin{array}{l}\text { 5. SIN MIEDO Airlines puede utilizarse en cualquier lugar y } \\
\text { en cualquier contexto. }\end{array}$ & $\begin{array}{c}3,25 \\
(0,96)\end{array}$ & 2 & 4 \\
\hline 6. Las instrucciones de SIN MIEDO Airlines son fáciles. & $\begin{array}{c}4 \\
(0,0)\end{array}$ & 4 & 4 \\
\hline $\begin{array}{l}\text { 7. El tamaño de letra y de los botones es suficiente para } \\
\text { mí. }\end{array}$ & $\begin{array}{c}4 \\
(0,0)\end{array}$ & 4 & 4 \\
\hline 8. Me gustaría utilizar este sistema frecuentemente. & $3,5(0,58)$ & 3 & 4 \\
\hline $\begin{array}{l}\text { 9. En general, creo que SIN MIEDO Airlines es muy útil para } \\
\text { mí. }\end{array}$ & $3,75(0,5)$ & 3 & 4 \\
\hline 10. En general, creo que SIN MIEDO Airlines es fácil de usar. & $3,75(0,5)$ & 3 & 4 \\
\hline
\end{tabular}

\section{Discusión y conclusiones}

El objetivo del presente trabajo era ofrecer datos sobre la usabilidad y aceptabilidad de un tratamiento autoaplicado a través de Internet para la FV (SIN MIEDO Airlines).

Los datos obtenidos a partir del estudio piloto reflejan una buena valoración de los participantes respecto a la usabilidad y aceptabilidad de SIN MIEDO Airlines. En concreto, los resultados revelaron puntuaciones altas en todos los aspectos de usabilidad y aceptabilidad valorados, como son: rapidez de aprendizaje, capacidad de uso, orientación, eficiencia, modelo ecológico, facilidad de las instrucciones, control y visibilidad, intención de uso, utilidad y facilidad percibida.

En este sentido, los resultados obtenidos en este estudio piloto sobre el programa SIN MIEDO Airlines podrían tener repercusiones en la mejora del tratamiento de la FV, especificamente en la mejora de la técnica de exposición, mejorando la aceptación del tratamiento y el acceso al mismo, al tratarse de un tratamiento autoaplicado a través de Internet. 
Estos resultados van en la línea de otros trabajos que ponen de manifiesto la utilidad de los programas asistidos por ordenador para la administración de la exposición en la FV (TorteIla-Feliu, Bornas y Llabrés, 2008; Tortella-Feliu y cols., 2011). No obstante, hasta la fecha, este es el primer estudio que se centra en explorar la usabilidad y aceptabilidad de un programa de tratamiento autoaplicado vía Internet para la FV.

A pesar de los prometedores resultados preliminares encontrados, el presente trabajo cuenta con una seríe de limitaciones. Al trarse de un estudio piloto, el tamaño muestral es pequeño $(\mathrm{N}=4)$, por lo que se requieren estudios con una mayor muestra para confirmar estos resultados preliminares. Por otra parte, en este estudio se ha evaluado la usabilidad y aceptabilidad, aunque no se han tenido en consideración las expectativas y la opinión de los participantes respecto al tratamiento. Este es un aspecto que forma parte del eje de eficiencia que debería tenerse en consideración en futuros estudios.

En conclusión, en este trabajo se han presentado datos sobre la usabilidad y aceptabilidad de SIN MIEDO Airlines que muestran la utilidad de este programa autoaplicado a través de Internet para la mejora de la técnica de exposición en el tratamiento de la FV. Es necesario llevar a cabo estudios de usabilidad y aceptabilidad para conseguir diseminar las TIC en el campo de la psicología clínica, especialmente entre los potenciales usuarios como son, en el caso del presente estudio, los pacientes. No obstante, en un futuro se requieren más investigaciones que sigan aportando evidencias a este respecto.

\section{Referencias bibliográficas}

American Psychiatric Association. (2013). Diagnostic and statistical manual of mental disorders (DSM-5®). Arlington VA, USA: American Psychiatric Association.

Andersson, G. (2016). Internet-Delivered Psychological Treatments. Annual Review of Clinical Psychology, 12, 157-179.

Andrews, G., Newby, J. M. y Williams, A. D. (2015). Internet-delivered cognitive behavior therapy for anxiety disorders is here to stay. Current psychiatry reports, 17, 1-5.

Bangor, A., Kortum, P. T. y Miller, J. (2009). Determining what individual sus scores mean: adding an adjective rating scale. Journal of Usability Studies, 4, 114-123.

Bangor, A., Kortum, P. T. y Miller J. T. (2008). An empirical evaluation of the system usability scale. International Journal of Human-Computer Interaction, 24, 574-594.

Baños, R., Botella, C., Perpiñá, C. y Quero, S. (2001). Tratamiento mediante realidad virtual para la fobia a volar: un estudio de caso. Clínica y Salud 12, 391-404.

Bor, R. (2007). Psychological factors in airline passenger and crew behaviour: a clinical overview. Travel Med Infect Dis, 5, 207-216.

Bornas, X., Fullana, M. A., Tortella-Feliu, M., Llabrés, J. y García de la Banda, G. (2001). Computer-assisted therapy in the treatment of flight phobia: a case report. Cognitive and Behavioral Practice, 8, 234-240.

Bornas, X., Tortella-Feliu, M., García de la Banda, G., Fullana, M. A. y Llabrés, J. (1999). Validación factorial del Cuestionario de Miedo a Volar. Análisis y Modificación de Conducta, 25, 885-907.

Botella, C., Osma, J., García-Palacios, A., Quero, S. y Baños, R. M. (2004). Treatment of flying phobia using virtual reality: data from a 1-year follow-up using a multiple baseline design. Clinical Psychology and psychotherapy, 11, 311-323.

Boyd, J. H., Rae, D. S., Thompson, J. W., Burns, B. J., Bourdon, K., Locke, B. Z. y Regier, D. A. (1990). Phobia: prevalence and risk factors. Social Psychiatrica and Psychiatric Epidemiology, 25, 314-323. 
Capafons, J. I. (2001). Tratamientos psicológicos eficaces para las fobias específicas. Psicothema, 13, 447-452.

Deacon, B. J. y Farrell, N. R. (2013). Therapist barriers to the dissemination of exposure therapy. En D. McKay y E. Storch (eds.), Handbook of Treating Variants and Complications in Anxiety Disorders (pp. 363-373). New York: Springer Press.

García-Palacios, A., Botella, C., Hoffman, H. y Fabregat, S. (2007).Comparing acceptance and refusal rates of virtual reality exposure vs. in vivo exposure by patients with specific phobias. Cyberpsychology \& Behavior, 10, 722-724.

García-Palacios, A., Hoffman, H. G., Kwong See, S., Tsai, A. y Botella, C. (2001). Redefining therapeutic success with virtual reality exposure therapy. CyberPsychology \& Behavior, 4, 341-348.

Iljon Foreman, E., Bor, R. y Van Gerwen, L. J. (2006). The nature, characteristics, impact and personal implications of fear of flying. En R. Bor y T. Hubbard (eds.) Aviation Mental Health (pp. 53-68). Aldershot: Ashgate.

Kazdin, A. E. (2014). Evidence-based psychotherapies II: changes in models of treatment and treatment delivery. South African Journal of Psychology. DOI: 10.1177/0081246314538733

Kazdin, A. E. y Blase, S. L. (2011). Rebooting psychotherapy research and practice to reduce the burden of mental illness. Perspectives on Psychological Science, 6, 21-37.

Kazdin, A. E. y Rabbitt, S. M. (2013). Novel models for delivering mental health services and reducing the burdens of mental illness. Clinical Psychological Science, 1, 170-191.

Konnopka, A., Leichsenring, F., Leibing, E. y König, H. H. (2009). Cost-of-illness studies and cost-effectiveness analyses in anxiety disorders: a systematic review. Journal of Affective Disorders, 114(1), 14-31.

Mackenzie, C. S., Reynolds, K., Cairney, J., Streiner, D. y Sareen, J. (2012). Disorder-specific mental health service use for mood and anxiety disorders: Associations with age, sex, and psychiatric comorbidity. Depression \& Anxiety, 29, 234-242.

Moritz, K., Hoffman, J., Herbert, J. D. y Schare, M. L. (2013, noviembre). Anxiety Disorders: Navigating Legal and Ethical Dilemmas. Paper presented at the $47^{\text {th }}$ Annual Convention of Cognitive and Behavioral Therapies (АВCT2013), Nashville. Abstract recuperado de http://abct2013.abstractcentral.com.

Nathan, P. y Gorman, J. (2007). A guide to treatments that work (3. ${ }^{a}$ ed.). New York: Oxford Press.

Oakes, M. y Bor, R. (2010). The psychology of fear of flying (part I): A critical evaluation of current perspectives on the nature, prevalence and etiology of fear of flying. Travel Medicine and Infectious Disease, 8, 327-338.

Olatunji, B., Deacon, B. J. y Abramowitz, J. S. (2009). The Cruelest Cure? Ethical Issues in the Implementation of Exposure-Based Treatments. Cognitive and Behavioral Practice, 16, 172-180.

Quero, S., Campos, D., Botella, C., Baños, R. M., García-Palacios., A. y Guillén, V. (2014, junio). Patients' Expectations and Satisfaction towards Traditional CBT versus CBT supported by ICTs. Poster presentado al 19th Annual CyberPsycholohy \& Cybertherapy Conference, Washington DC, USA.

Quero, S., Campos, D., Riera del Amo, A., Bretón, J., Tortella, M., Baños, R. y Botella, C. (2015). No-FEAR Airlines: a computer-aided self-help treatment for flying phobia. Studies in Health Technology and Informatics, 219, 197-201.

Richard, D. C. S. y Gloster, A. T. (2007). Exposure therapy has a public relations problem: A dearth of litigation amid a wealth of concern. Comprehensive handbook of the exposure therapies, 409-425. New York: Academic Press. 
Rothbaum, B. O., Hodges, L., Smith, S., Lee, J. H. y Price, L. (2000). A controlled study of virtual reality exposure therapy for the fear of flying. Journal of consulting and Clinical Psychology, 68, 1020.

Stinson, F. S., Dawson, D. S., Chou, S. P., Smith, S., Goldstein, R. B., Ruan, W. J. y Grant, B. F. (2007). The epidemiology of DSM-IV specific phobia in the USA: Results from the national epidemiologic survey on alcohol and related conditions. Psychological Medicine, 37, 1047-1059.

Tortella-Feliu, M., Bornas, X. y Llabrés, J. (2008). Computer-Assisted Exposure Treatment for Flight Phobia. International Journal of Behavioral Consultation and Therapy, 4, 158-71.

Tortella-Feliu, M., Botella, C., Llabrés, J., Bretón-López, J. M., Riera del Amo, A., Baños, R. M. y Gelabert, J. M. (2011). Virtual Reality Versus Computer-Aided Exposure Treatments for Fear of Flying, Behavior Modification, 35, 3-30. 